\section{K/Ca-Altersbestimmungen an Kalifeldspäten}

\author{
Klaus G. Heumann, Werner Schwabenbauer \\ und Irene Stadler \\ Institut für Chemie der Universität Regensburg \\ und Emil Kubassek \\ Fachbereich Anorganische Chemie und Kernchemie \\ der TH Darmstadt
}

(Z. Naturforsch. 32 a, 1333-1334 [1977] ; eingegangen am 20. Oktober 1977)

\section{Potassium/Calcium Age Determination, Feldspars, Mass Spectrometry}

A method for the potassium/calcium age determination of geological material is described. The calcium isotope intensities are measured with a mass spectrometer by the thermal ionization technique. The ${ }^{42} \mathrm{Ca} /{ }^{44} \mathrm{Ca}$ isotope ratio is used as an internal standard to correct the measured ${ }^{40} \mathrm{Ca} /{ }^{42} \mathrm{Ca}$ ratio for mass discrimination. The radiogenic ${ }^{40} \mathrm{Ca}$ portion and the calcium content of the minerals are determined in different analytical steps. Two feldspars are dated with relative standard deviations of 1.8 and 4 per cent, respectively.

Von den gegenwärtig existierenden Datierungsmöglichkeiten für geologische Proben werden die $\mathrm{K} / \mathrm{Ar}$-, $\mathrm{Rb} / \mathrm{Sr}$ - und $\mathrm{U} / \mathrm{He}(\mathrm{Pb})$-Methoden am häufigsten angewandt ${ }^{1-3}$. In vielen Fällen ist es sinnvoll, weitere Alternativen zur Verfügung zu haben. So wurden mit der $\mathrm{K} / \mathrm{Ca}$-Datierung bisher nur einige Sylvine $^{4,5}$ und einige Glimmerarten ${ }^{6,7}$ bestimmt. Dies ist vor allen Dingen auf die Schwierigkeit zurückzuführen, den radiogenen ${ }^{40} \mathrm{Ca}$-Anteil analytisch zu ermitteln. Da uns in den letzten Jahren eine wesentliche Verbesserung der Reproduzierbarkeit der Isotopenverhältnismessungen des Calciums im Massenspektrometer gegenüber bisherigen Literaturangaben gelungen ist ${ }^{8}$, haben wir ein analytisches Verfahren zur K/Ca-Datierung entwickelt. Dieses ist auch auf andere Mineralien als die zuvor genannten anwendbar, wobei vor allem die Datierung der häufig vorkommenden Feldspäte von Interesse ist.

\section{K/Ca-Altersgleichung}

Natürliches Kalium enthält zu 0,0119\% das radioaktive ${ }^{40} \mathrm{~K}$-Isotop ${ }^{9} .{ }^{40} \mathrm{~K}$ zerfällt unter Elektroneneinfang $(\varepsilon)$ mit etwa $11 \%$ Häufigkeit in ${ }^{40} \mathrm{Ar}$ und unter $\beta^{-}$-Strahlung mit ungefähr $89 \%$ der Zerfälle in ${ }^{40} \mathrm{Ca}^{10}$ :

$$
{ }^{40} \mathrm{~K} \nearrow_{\beta^{-}}^{\varepsilon}{ }^{40} \mathrm{Ca} \text { (radiogenes }{ }^{40} \mathrm{Ca} \text { ) }
$$

Sonderdruckanforderungen an Prof. Dr. K. G. Heumann, Institut für Chemie der Universität, Universitätsstraße 31, D-8400 Regensburg.
Bestimmt man nun den relativen, radiogenen ${ }^{40} \mathrm{Ca}$ Anteil $\left[\left({ }^{40} \mathrm{Ca} /{ }^{42} \mathrm{Ca}\right)_{\mathrm{t}}-\left({ }^{40} \mathrm{Ca} /{ }^{42} \mathrm{Ca}\right)_{\mathrm{akz}}\right]$ sowie den Ca- und K-Gehalt der Probe, so kann man nach Gl. (2) das Alter $t$ berechnen:

$$
\begin{aligned}
t=\frac{1}{\lambda_{\varepsilon}+\lambda_{\beta}} \cdot \ln \{ & 1+\frac{\left(\lambda_{\varepsilon}+\lambda_{\beta}\right)}{\lambda_{\beta}} \cdot \frac{N_{\mathrm{Ca}} \cdot \mathrm{h}^{42} \mathrm{Ca}}{N_{\mathrm{K}} \cdot \mathrm{h}^{40 \mathrm{~K}}} \\
& \left.\cdot\left[\left({ }^{40} \mathrm{Ca} /{ }^{42} \mathrm{Ca}\right)_{\mathrm{t}}-\left({ }^{40} \mathrm{Ca} /{ }^{42} \mathrm{Ca}\right)_{\mathrm{akz}}\right]\right\} ;
\end{aligned}
$$

$\lambda_{\varepsilon, \beta}$ Zerfallskonstante des ${ }^{40} \mathrm{~K}$ für den $\varepsilon$ - bzw. $\beta^{-}$-Zerfall; $N_{\mathrm{Ca}, \mathrm{K}}$ Zahl der Ca- bzw. K-Atome in der Mineralprobe; $\quad\left({ }^{40} \mathrm{Ca} /{ }^{42} \mathrm{Ca}\right)_{\mathrm{t}, \mathrm{akz}}$ Isotopenverhältnis ${ }^{40} \mathrm{Ca} /{ }^{42} \mathrm{Ca}$ im Mineral bzw. des akzessorischen Calciums; $\mathrm{h}_{42} \mathrm{Ca}$ Häufigkeit von ${ }^{42} \mathrm{Ca}$ im Mineral [\%]; $\mathrm{h}_{40 \mathrm{~K}}$ Häufigkeit von ${ }^{40} \mathrm{~K}$ im natürlichen Kalium [\%].

\section{Experimentelles}

Bei unseren Analysen wurde der relative, radiogene ${ }^{40} \mathrm{Ca}$-Anteil nach Aufschluß der Probe und Separation des Calciums über eine chromatographische Säule durch Messung der Isotopenintensitäten des ${ }^{40} \mathrm{Ca},{ }^{42} \mathrm{Ca}$ und ${ }^{44} \mathrm{Ca}$ in einem Thermionen-Massenspektrometer bestimmt. Da Calcium mehrere stabile, nicht radiogene Isotope besitzt, kann das gemessene ${ }^{42} \mathrm{Ca} /{ }^{44} \mathrm{Ca}$-Verhältnis als interner Standard zur Kor-

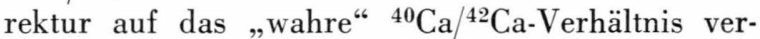
wendet werden ${ }^{11}$. Diese Korrektur ist notwendig, um die unvermeidbare Massendiskriminierung bei der massenspektrometrischen Messung zu kompensieren. Als „wahres“ (= natürliches) ${ }^{42} \mathrm{Ca} /{ }^{44} \mathrm{Ca}-\mathrm{Ver}$ hältnis wurde dabei der Wert 0,3125 zugrunde gelegt ${ }^{12}$. Es wurde außerdem angenommen, daß $\left({ }^{40} \mathrm{Ca} /{ }^{42} \mathrm{Ca}\right)_{\text {akz }}$ mit dem ${ }^{40} \mathrm{Ca} /{ }^{42} \mathrm{Ca}-$ Verhältnis des natürlichen Calciums übereinstimmt. In weiteren Aufschlüssen wurde der Calciumgehalt der Proben durch massenspektrometrische Isotopenverdünnungsanalyse mit einem ${ }^{42} \mathrm{Ca}$-Indikator ${ }^{13} \mathrm{bzw}$. der K-Gehalt durch massenspektrometrische Isotopenverdünnungsanalyse mit einem ${ }^{41} \mathrm{~K}$-Indikator oder durch Flammen-Atomemissionsspektrometrie mit Lithium als Leitelement ${ }^{14}$ bestimmt.

\section{Ergebnisse und Diskussion}

Die Ergebnisse der Analysen eines Amazonits (Gordonia-Distrikt/Süd-Afrika) und eines Mikroklins (Namaqua-Mobile-Belt/Südwest-Afrika) ${ }^{*}$ sind in Tab. 1 zusammengefaßt. Dabei beziehen sich die Fehlerangaben (Wiederholungs-Standardabweichungen) auf jeweils 3 voneinander unabhängig durch-

\footnotetext{
* Herrn Prof. Dr. A. Kröner, Geologisches Institut der Universität Mainz, danken wir für die Überlassung der Proben.
} 


\begin{tabular}{lllc}
\hline Probe & $\left({ }^{40} \mathrm{Ca} /{ }^{42} \mathrm{Ca}\right) \mathrm{t}$ & $\begin{array}{l}\text { K/Ca-Alter } \\
\text { [Jahre] }\end{array}$ & $\begin{array}{c}S_{\text {rel. }}(\mathrm{K} / \text { Ca-Alter }) \\
{[\%]}\end{array}$ \\
\hline Amazonit & $153,66 \pm 0,08$ & $(1,10 \pm 0,02) \cdot 10^{9}$ & 1,8 \\
Mikroklin & $151,91 \pm 0,19$ & $(2,25 \pm 0,09) \cdot 10^{9}$ & 4,0 \\
natürliches & & & \\
Calcium $^{12}$ & 149,14 & & \\
\hline
\end{tabular}

Tab. 1. Radiogener ${ }^{40} \mathrm{Ca}-$ Anteil und $\mathrm{K} /$ Ca-Alter von Kalifeldspäten. geführte Aufschlüsse zur Bestimmung des relativen, radiogenen ${ }^{40} \mathrm{Ca}$-Anteils. Nach Gl. (2) geht die Differenzbildung $\left[\left({ }^{40} \mathrm{Ca} /{ }^{42} \mathrm{Ca}\right)_{\mathrm{t}}-\left({ }^{40} \mathrm{Ca} /{ }^{42} \mathrm{Ca}\right)_{\mathrm{akz}}\right]$, da sie von zwei ähnlich großen Zahlen gebildet wird, gegenüber Ungenauigkeiten bei der Ermittlung des Ca- und K-Gehalts in größerem Maße in den Fehler der Altersdatierung ein. In beiden Feldspäten ist eine deutliche Abweichung des ${ }^{40} \mathrm{Ca} /{ }^{42} \mathrm{Ca}$-Verhältnisses gegenüber natürlichem Calcium zu verzeichnen, woraus sich das $\mathrm{K} / \mathrm{Ca}$-Alter des Amazonits zu $1,10 \cdot 10^{9}$, dasjenige des Mikroklins zu $2,25 \cdot 10^{9} \mathrm{Jah}$ ren ergibt. Damit ist es erstmalig gelungen, eine $\mathrm{K} / \mathrm{Ca}$-Datierung auch an Feldspäten durchzuführen.

Noch vor wenigen Jahren glaubte man ${ }^{15}$, daß die „K/Ca-Uhr" nicht brauchbar ist, da es sich praktisch als unmöglich erwies, die kleine radiogene Ca-Komponente vom primären Calcium zu unterscheiden. Unsere Analysen haben jedoch gezeigt, daß dies grundsätzlich möglich ist. Voraussetzungen für die Anwendbarkeit der K/Ca-Methode sind in der Regel $\mathrm{K} / \mathrm{Ca}$-Verhältnisse in der Probe von $>50$ (Mikro-

${ }^{1}$ L. T. Aldrich, G. L. Davis, G. R. Tilton, G. W. Wetherill u. P. M. Jeffrey, Nuclear Process in Geologic Settings, Nuclear Science Series Report No. 19, National Research Council, Washington D. C. 1956.

2 G. J. Wasserburg u. R. J. Hayden, Geochim. Cosmochim. Acta 7, 51 [1955].

3 G. B. Dalrymple u. M. A. Lanphere, Potassium-Argon Dating, Freeman and Co., San Francisco 1969.

4 N. I. Polevaya, N. E. Titov, V. S. Belyaev u. V. D. Sprintsson, Geochemistry USSR, English Transl. 8, 897 [1958].

5 H. G. Wilhelm u. W. Ackermann, Z. Naturforsch. 27 a, 1256 [1972].

6 M. M. Backus, Dissertation, Massachusetts Institute of Technology, Massachusetts 1955.

7 M. L. Coleman, Earth Planet. Sci. Lett. 12, 399 [1971]. klin: $\mathrm{K} / \mathrm{Ca} \approx 72 ;$ Amazonit: $\mathrm{K} / \mathrm{Ca} \approx 322$ ) sowie Alter von $>10^{8}$ Jahren. Entscheidend für die K/CaDatierung an Feldspäten war die Verbesserung des gesamten analytischen Verfahrens. Hierbei war mit ausschlaggebend, daß wir den radiogenen ${ }^{40} \mathrm{Ca}-\mathrm{An}$ teil und den Ca-Gehalt der Probe nicht in Analogie zu der sonst üblichen Vorgehensweise in einem Schritt durch Isotopenverdünnung bestimmt haben, sondern diese beiden Größen in unabhängigen Analysengängen ermittelten. Die relativen WiederholungsStandardabweichungen $s_{\text {rel. }}$ von 1,8 bzw. $4 \%$ zeigen die gute Reproduzierbarkeit unseres analytischen Verfahrens. Inwieweit die K/Ca-Alter mit den Ergebnissen anderer Datierungsmethoden vergleichbar sind, sollen künftige Bestimmungen von Proben zeigen, deren Alter auch auf andere Weise ermittelt wurden.

Der Deutschen Forschungsgemeinschaft und dem Fonds der Chemischen Industrie danken wir für die Unterstützung dieser Arbeit.

${ }^{8}$ K. G. Heumann u. E. Kubassek, Advances in Mass Spectrometry, Vol. 6, p. 611, Applied Science Publishers Ltd., Barking, Essex 1974.

9 A. O. Nier, Phys. Rev. 77, 789 [1950].

10 L. T. Aldrich u. G. W. Wetherill, Ann. Rev. Nucl. Sci. 8, 257 [1958].

11 M. H. Dodsen, J. Sci. Instrum. 40, 289 [1969].

12 W. Seelmann-Eggebert, G. Pfennig u. H. Münzel, Nuklidkarte, 4. Aufl., Gersbach u. Sohn Verlag, München 1974.

13 K. G. Heumann, E. Kubassek u. W. Schwabenbauer, Z. Anal. Chem. 287, 121 (1977).

14 E. Kubassek u. K. G. Heumann, Z. Anal. Chem., im Druck.

15 F. Gönnenwein, Physik in unserer Zeit 3, 81 [1972]. 